\title{
PROPUESTA PARA LA OPTIMIZACIÓN DEL DISEÑO Y OPERACIÓN DE SISTEMAS FOTOVOLTAICOS AUTÓNOMOS A INSTALARSE EN EL PERÚ, BASADO EN REDES ELÉCTRICAS INTELIGENTES

\author{
PROPOSSAL FOR THE OPTIMIZATION DESIGN AND \\ OPERATION OF AUTONOMOUS PHOTOVOLTAIC SYSTEMS \\ INSTALLED IN PERU BASED ON SMARTGRIDS
}

\begin{abstract}
Oswaldo Morla Morales Taquiri ${ }^{1}$
RESUMEN

El cambio climático obliga a un crecimiento económico sin contaminación. Una de las principales alternativas es el uso de energías renovables como la solar fotovoltaica. La variabilidad del recurso solar, de la demanda y de las condiciones ambientales que incidirán en su performance. Para el buen funcionamiento de los sistemas fotovoltaicos, autónomos, es indispensable desarrollar una metodología de optimización de su diseño y operación, autónomos, que tomen en cuenta la oferta, demanda y las condiciones ambientales, utilizando para ello criterios de redes eléctricas inteligentes. Este trabajo propone la monitorización y predicción del rendimiento de los sistemas fotovoltaicos, así como el fomento de estrategias de planificación y control de modo eficiente, utilizando un regulador seguidor del punto de máxima potencia, una estación meteorológica que incluya la medición de polvo, un sistema de adquisición de datos inalámbrica y una estación de monitoreo y registro basado en LabVIEW.
\end{abstract}

Palabras clave.- Optimización de sistemas fotovoltaicos, sistemas fotovoltaicos autónomos, LabVIEW en sistemas fotovoltaicos.

\begin{abstract}
Climate change forces economic growth without pollution. One of the main alternatives is the use of renewable energy such as solar photovoltaic. The variability of the solar resource, the demand and the environmental conditions that will affect its performance. For the proper functioning of autonomous photovoltaic systems, it is indispensable to develop a methodology for optimizing its design and operation, autonomous, taking into account the supply, demand and environmental conditions, using criteria for intelligent electric grids. This project proposes the monitoring and prediction of the performance of photovoltaic systems, as well as the promotion of efficient planning and control strategies. It is proposed to use a maximum power point tracking controller, a weather station that includes dust measurement, a wireless data acquisition system, and a LabVIEW based monitoring and registration station
\end{abstract}

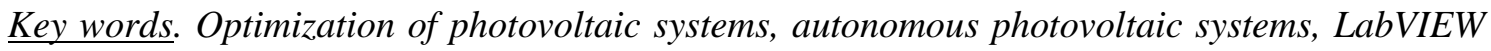
in photovoltaic systems.

\footnotetext{
La Revista Científica TECNIA protege los derechos de autor bajo la Licencia 4.0 de Creative Commons: Attribution 4.0 International (CC BY 4.0)

${ }^{1}$ Facultad de Ingeniería Mecánica, Universidad Nacional de Ingeniería, Av. Túpac Amaru N ${ }^{\circ}$

210 Rímac, Lima-Perú
} 


\section{INTRODUCCIÓN}

Debido al cambio climático, el crecimiento económico sin contaminación es uno de los principales problemas que debe resolverse en las próximas décadas. [1-7].

Las instalaciones fotovoltaicas están aumentando de manera constante. Estos sistemas, sin embargo, no pueden trabajar de manera eficiente si su operación no está automatizada [8].

Asimismo, la necesidad de mejorar el rendimiento de estos sistemas de energía en términos de fiabilidad y mayor productividad ha requerido cada vez más de aplicaciones técnicas de supervisión. [8-9].

Por lo tanto, el diseño y operación de cualquier sistema fotovoltaico autónomo debería utilizar una metodología de optimización, tomando en cuenta la oferta, demanda y condiciones ambientales, basado en criterios de redes eléctricas inteligentes, tema que este proyecto desarrollará.

Las posibles entidades interesadas en los resultados de este trabajo son:

- Ministerios de: Energía y Minas, Educación, Salud, Ambiente.

- Osinergmin, Adinelsa.

- Gobiernos Regionales, Municipalidades.

- Bancos Nacionales y Extranjeros.

- ONG's.

- Empresas comercializadoras de: Módulos Fotovoltaicos, Reguladores con seguimiento de máxima potencia, Inversores, y Sistemas de Monitoreo y Control.

- Proveedores de software.

- Centros de investigación en energías renovables.

\section{MOTIVACIÓN Y JUSTIFICACIÓN}

Los sistemas fotovoltaicos son actualmente la mejor alternativa para satisfacer la demanda de energía eléctrica. Estos sistemas pueden ser instalados dentro de una red eléctrica o en forma autónoma [14-16]. En ambos casos para mejorar su rendimiento, fiabilidad y productividad $\mathrm{su}$ operación debe ser automatizada.

Por lo tanto, existe la necesidad del desarrollo de una metodología para la optimización de sistemas fotovoltaicos autónomos a instalarse en el Perú utilizando el criterio de redes eléctricas inteligentes.

Las aplicaciones de control y automatización tienen como finalidad desarrollar soluciones para reducir costos y mejorar la calidad del servicio.

En este proyecto se desarrollará una metodología para optimizar el diseño y operación de sistemas fotovoltaicos, tomando en cuenta la oferta, demanda y condiciones ambientales, basado en criterios de redes eléctricas inteligentes [8-13].

\section{ANTECEDENTES}

Los módulos fotovoltaicos que actualmente se están instalando en el Perú son fundamentalmente de Silicio; monocristalino, policristalino y amorfo o de película delgada, cuyas necesidades de áreas para instalar $1 \mathrm{kWp}$ se encuentra en la figura 1 .

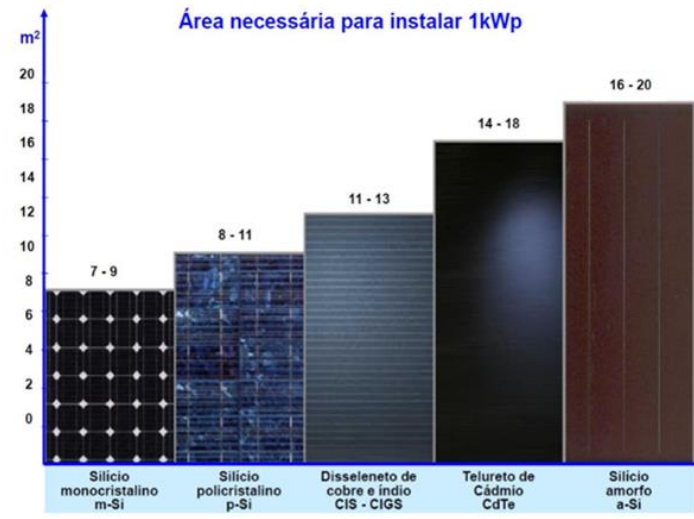

Figura 1: Área de módulos fotovoltaicos para instalar 1kWp (Fuente: Dr. Clodomiro Unsihuay Vila. 2015)

Las eficiencias de las células fotovoltaicas dependen de la forma de cristalización, siendo las de monocristalización las más eficientes y las de cristalización amorfa o de película delgada menor eficiencia. En la figura 2 se muestran los valores de estas eficiencias. 


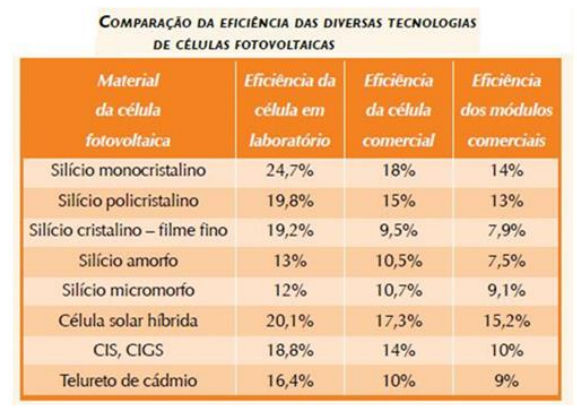

Figura 2: Eficiencias de las células fotovoltaicas. Fuente: Dr. Clodomiro Unsihuay Vila. 2015.

Para cualquier diseño con sistemas fotovoltaicos es importante conocer la curva característica del módulo fotovoltaico, pues su potencia de salida depende de la carga, la radiación solar y de la temperatura, tal como se muestra en la figura 3.

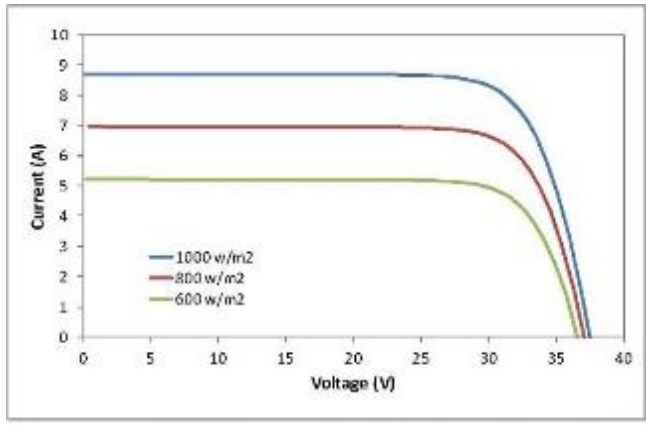

Figura Fa. Curvas I-V para distintos valores de is irradiancia solar Temperatura constante de la célula $\left(25^{\circ} \mathrm{C}\right)$

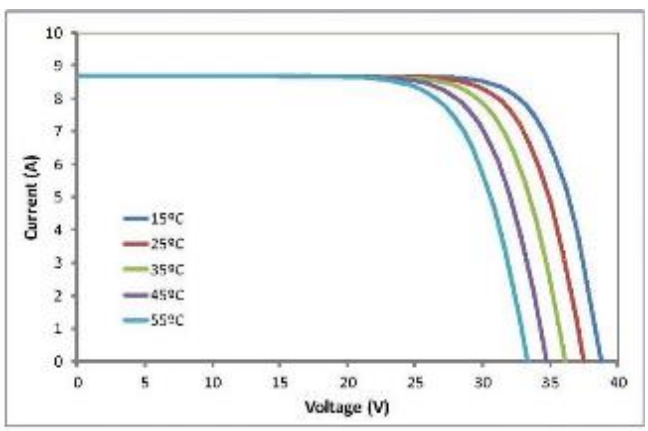

Figura Fb. Curvas I-V para distintas temperatura de la célula Valor de irradiancia solar constante $\left[1000 \mathrm{~W}^{2} \mathrm{~m}^{2}\right\}$

Figura 3: Curvas características de un módulo fotovoltaico vs la radiación y la temperatura

Fuente: Google. Conversión Fotovoltaica. Imágenes

La dependencia de la corriente, potencia y voltaje versus la temperatura, se muestra en la figura 4 .

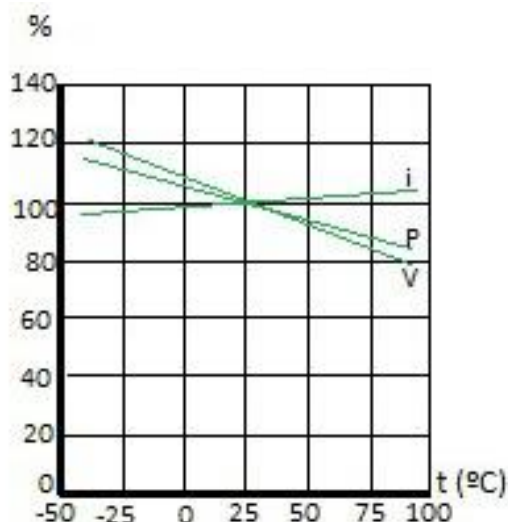

Figura 4: Corriente, Potencia y Voltaje versus Temperatura.

Fuente: Google. Conversión Fotovoltaica. Imágenes.

En la figura 5 se muestra la respuesta del módulo fotovoltaico a diferentes niveles de radiación solar.

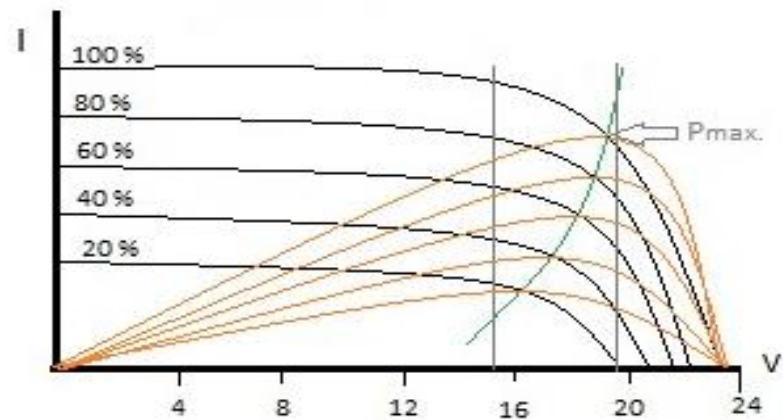

Figura 5: Funcionamiento de un módulo fotovoltaico en el rango de potencia nominal.

Fuente: Google. Conversión Fotovoltaica. Imágenes

La máxima potencia que entrega un módulo fotovoltaico se da cuando trabaja en el punto de potencia máxima o nominal. En la actualidad existen reguladores para sistemas fotovoltaicos seguidores del punto de máxima potencia (MPPT).

Los parámetros a evaluar en los módulos fotovoltaicos son: la potencia, el factor de forma, la eficiencia, el coeficiente de rendimiento y el factor de capacidad.

\section{Potencia:}

$P_{p v}=I_{T} \times A_{p v} \times \eta_{p v}$

Donde: 
$P_{p v}$ : Potencia del panel fotovoltaico (W)

IT: Radiación Total $\left(\mathrm{W} / \mathrm{m}^{2}\right)$

$\mathrm{A}_{\mathrm{pv}}$ : Área del panel fotovoltaico $\left(\mathrm{m}^{2}\right)$

$\boldsymbol{\eta}_{\mathrm{pv}}$ : Eficiencia del panel fotovoltaico a potencia pico.

Factor de forma (FF):

$F F_{p v}=\frac{V_{\text {nom }} \times I_{\text {nom }}}{V_{c a} \times I_{c c}}$

Donde:

$\mathrm{V}_{\text {nom: }}$ : Voltaje nominal (Volt)

$\mathrm{I}_{\text {nom: }}$ Corriente nominal (Amper)

$\mathrm{V}_{\text {ca: }}$ Voltaje de circuito abierto (Volt)

$\mathrm{I}_{\mathrm{cc}}$ : Corriente de corto circuito (Amper)

Eficiencia del módulo a potencia pico:

$\eta_{p v}=\frac{V_{n o m} \times I_{n o m}}{I_{T} \times A_{p v}}$

\section{Coeficiente de Rendimiento (CR):}

$C R_{p v}=\frac{\text { Energía Generada Real }}{\text { Energia Generada Teóricamente }}$

\section{Factor de Capacidad (FC):}

$F C_{p v}=\frac{\text { Energía Generada Real }}{\text { Potencia } \times \text { Horas en el año }}$

La evaluación de estos parámetros en forma constante permitirá conocer el estado en que se encuentran los módulos fotovoltaicos.

\section{PROBLEMATICA DE LOS SISTEMAS FOTOVOLTAICOS}

a) La calidad del cristal que se emplea como cobertor de los módulos fotovoltaicos influye en la eficiencia de éstos durante la absorción de la potencia emisiva de la radiación solar, cuyo valor de energía permitirá pasar a los electrones de la banda de valencia a la banda de conducción. En la figura 6 se muestra dos tipos de cristal de diferente calidad.

\section{TRANSMISIÓN DEL CRISTAL}

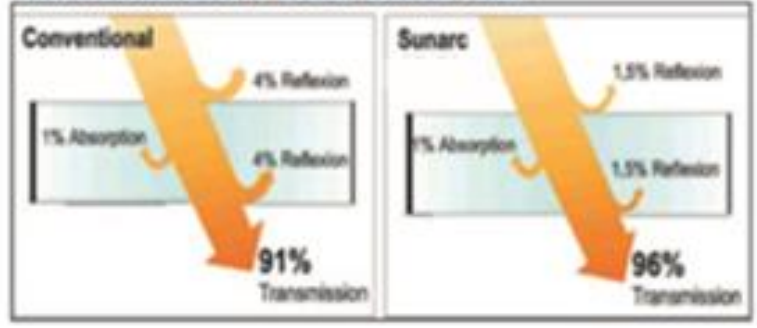

Figura 6: Calidad del cristal que cubre el módulo fotovoltaico.

Fuente: Catálogo Hispania Solar

b) En el mercado existen módulos fotovoltaicos de diferente calidad. Un módulo fotovoltaico estándar sólo garantiza el $90 \%$ de su potencia nominal al inicio de su instalación. Esta potencia de generación disminuye en el tiempo y al cabo de 10 años el módulo sólo genera el $80 \%$ de su capacidad nominal. En la figura 7 se muestra el rendimiento de dos tipos de módulos fotovoltaicos.

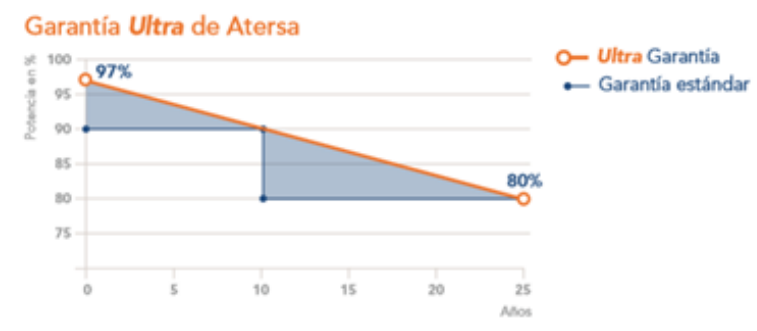

Figura 7: Variación de la eficiencia versus el tiempo de vida de los módulos fotovoltaicos.

Fuente: Energía fotovoltaica ATERSA

c) Adicionalmente, el módulo fotovoltaico durante su tiempo de vida útil se ve afectado por las inclemencias del clima, como la temperatura, humedad, polvo, precipitación pluvial, sombras que originan el efecto "disco caliente", etc.

d) Los sistemas fotovoltaicos son diseñados con suposiciones de demanda, oferta y recursos de energía solar no confiables, lo que origina que en la realidad durante la operación este sistema esté sobredimensionado o subdimensionado, lo que origina en el primer 
caso un mayor costo de instalación y en el segundo caso un déficit de entrega de energía.

e) Además, por la naturaleza del recurso energético que es variable y de la demanda que también es variable, tanto diario como estacional se hace necesario la existencia de una metodología para la optimización de sistemas fotovoltaicos autónomos a instalarse en el Perú, utilizando el criterio de red eléctrica inteligente, lo cual va a permitir el mejor funcionamiento del sistema en términos de costo, confiabilidad y eficiencia [14-25]. En la figura 8 se muestra la variabilidad del recurso solar mensual en el Perú.
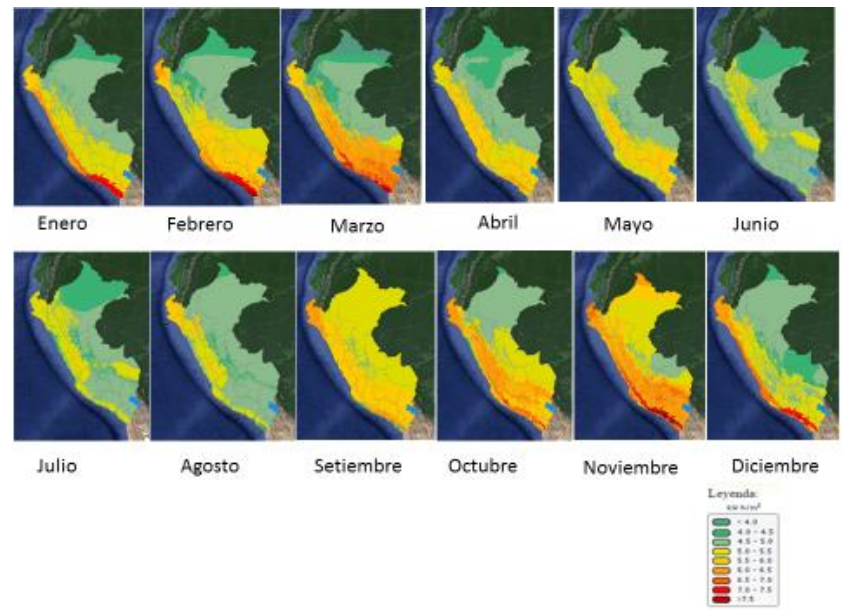

Figura 8: Energía solar $\left(\mathrm{kWh} / \mathrm{m}^{2} /\right.$ día) promedio mensual en el Perú.

Fuente: Atlas Solar del Perú 2003. MINEM

En la figura 9 se muestra las medias mensuales y anual de radiación solar media diaria, dadas por cuatro entidades: Atlas SWERA, NASA, Sundata y Atlas-UFPE, incidente sobre un colector inclinado de $10^{\circ} \mathrm{N}$ en la región de Manaos-Brasil. Una media de cada serie está mostrada por un símbolo sobre el eje vertical en el "mes cero". Cada serie es proveniente de un banco de datos diferente, lo que refuerza lo expresado en el párrafo anterior respecto a la necesidad de contar con una estación meteorológica.

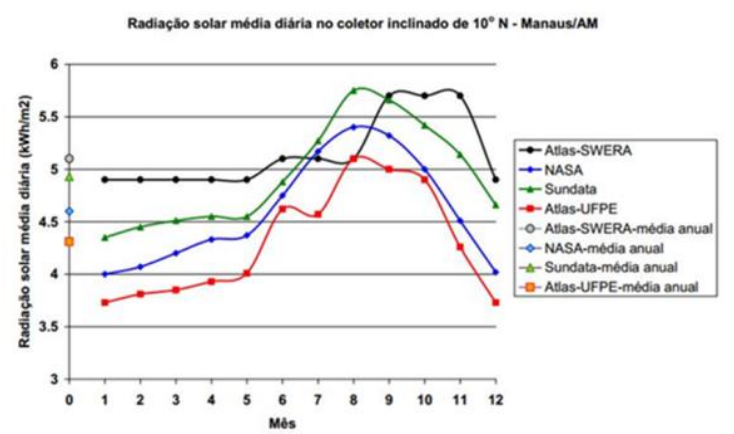

Figura 9: Radiación solar media diaria en colector inclinado $10^{\circ} \mathrm{N}$. Manaos-Brasil

Fuente: SOARES et al. 2010

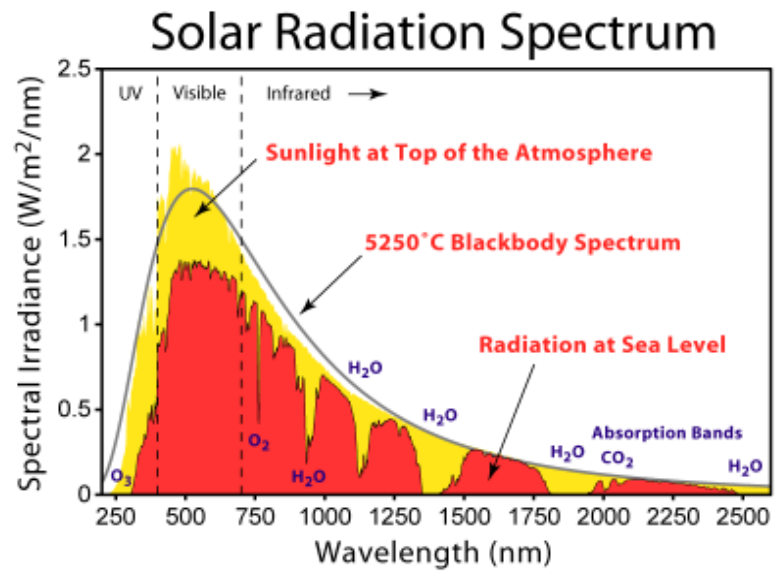

Figura 10: Potencia emisiva de la radiación solar $\left(\mathrm{W} / \mathrm{m}^{2} / \mathrm{nm}\right)$ versus la longitud de onda $(\mathrm{nm})$.

Fuente: Google. Radiación solar. Imágenes

f) La potencia emisiva de la radiación solar $\left(\mathrm{W} / \mathrm{m}^{2} / \mathrm{nm}\right)$, tal como se muestra en la figura 10, que incide sobre el territorio peruano, debido al cambio climático, ya no depende sólo de la presencia de agua en la atmósfera, sino también de los atenuadores o filtros que se encuentran en ella, como aerosoles, dióxido de carbono, nitrógeno, polvo, etc.

g) Actualmente los patrones diarios de radiación solar se han clasificado en cuatro clases: cielo claro, cielo claro intermitente, cielo completamente nublado y cielo con nubes intermitente. Esta clasificación es relevante para fines de análisis y modelación. Esta toma de datos permite la estimación de la radiación solar que se espera que se mide en un punto de medida dado, evitando el uso de expresiones complicadas como la altura del sol, el albedo, la transparencia atmosférica y la nubosidad [26]. 
A continuación, en las figuras (11-14), se presenta la clasificación de los patrones diarios de la radiación solar [26]:

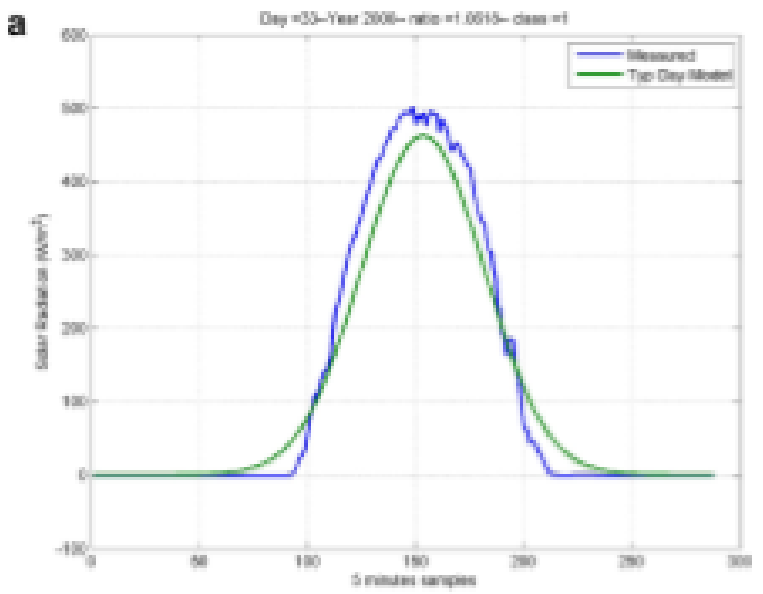

Figura 11: Clase 1: Cielo limpio, días de radiación solar con muy pocas nubes.

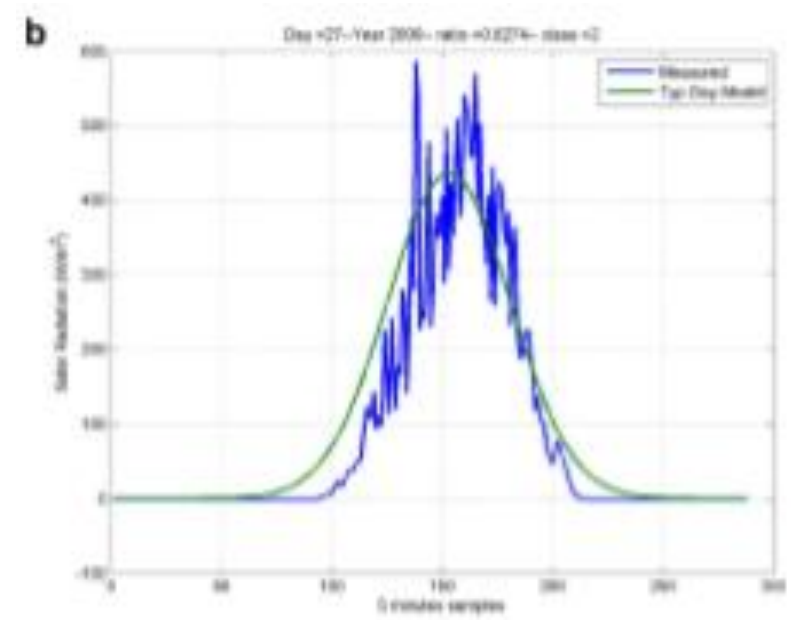

Figura 12: Clase 2: Cielo despejado intermitente, caracterizado por una importante radiación solar con algunas nubes, correspondiente a un nivel medio dinámico.

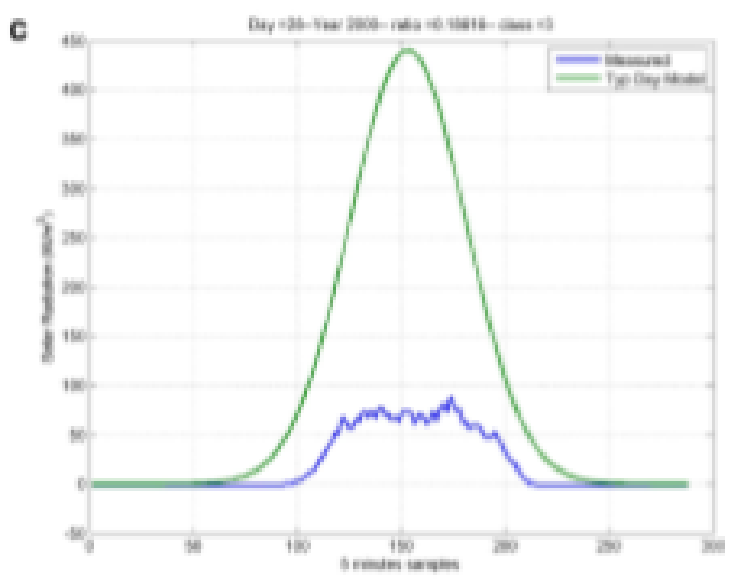

Figura 13: Clase 3: Cielo nublado, días de cielo completamente nublado, con grandes nubes con baja velocidad de movimiento, donde tanto la intensidad de radiación solar como el nivel dinámico es débil.

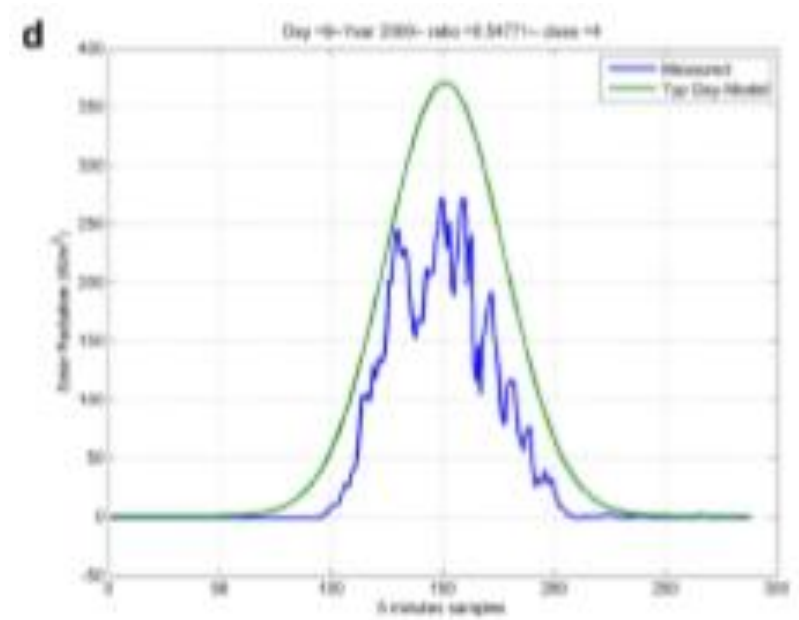

Figura 14: Clase 4: Cielo nublado intermitente, días con significativo brillo solar combinado con un gran número de pequeñas nubes con alta velocidad de movimiento, por consiguiente, con un nivel altamente dinámico.

h) Los módulos fotovoltaicos están expuestos a la radiación solar, temperatura, humedad relativa, lluvia y polvo del medio ambiente. La acumulación de polvo en los módulos fotovoltaicos es un factor degradante primordial. La suciedad y su efecto sobre el rendimiento de los módulos fotovoltaicos son de alta preocupación en las regiones con una alta deposición de polvo y baja frecuencia y menor intensidad de lluvias. Sin embargo, algunas zonas con precipitaciones abundantes 
también pueden sufrir de alta deposición de polvo en la estación seca. En este estudio propone investigar el efecto del polvo y de las otras condiciones meteorológicas en la performance de los módulos fotovoltaicos instalados en un determinado lugar, con el objetivo de calcular una ecuación de regresión que describa la pérdida de eficiencia [27].

Como ejemplo, en las figuras (15-22), se muestra los efectos de la acumulación de polvo y de las condiciones ambientales en la eficiencia de los módulos fotovoltaicos solares instalados fuera de la red en Kathmandu, Nepal [27].

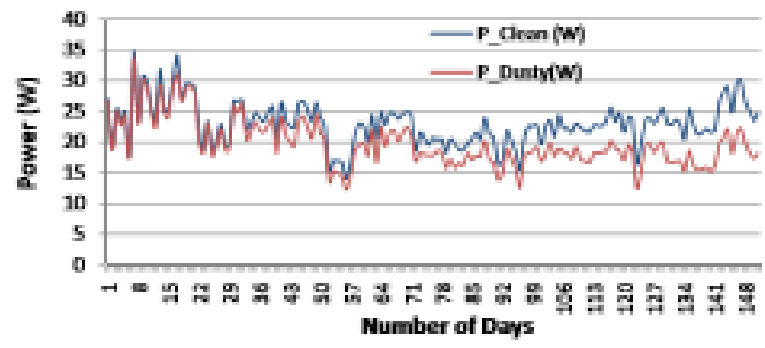

Figura 15: Potencia de salida de los módulos fotovoltaicos limpios y con polvo

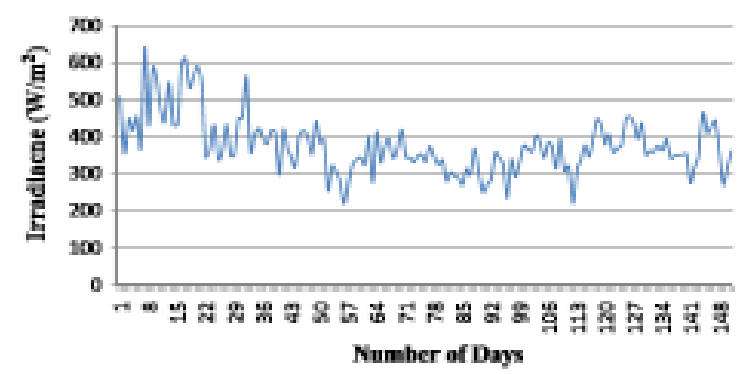

Figura 16: Medición de la radiación solar global sobre una superficie horizontal

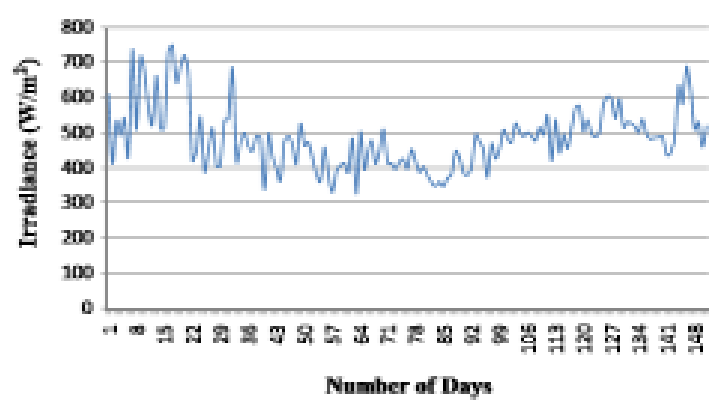

Figura 17: Medición de la radiación solar global sobre una superficie inclinada

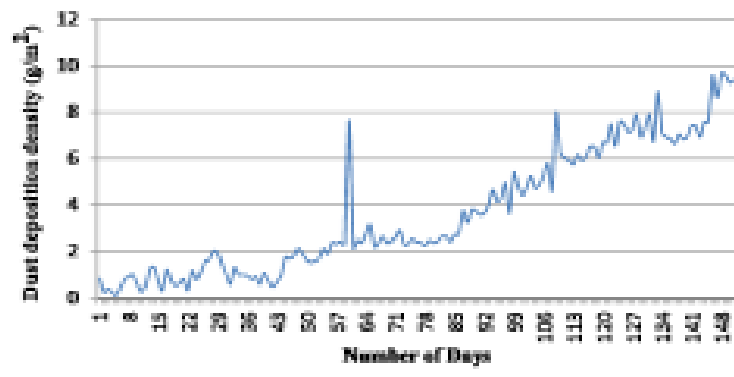

Figura 18: Medida de la densidad de deposición de polvo

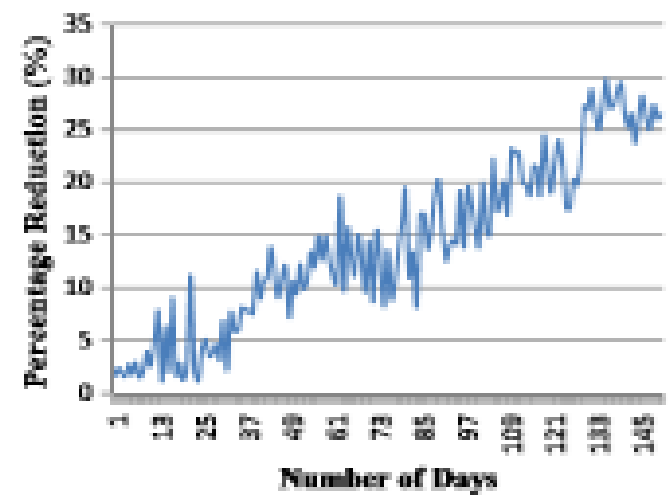

Figura 19: Porcentaje de la reducción de eficiencia del módulo fotovoltaico

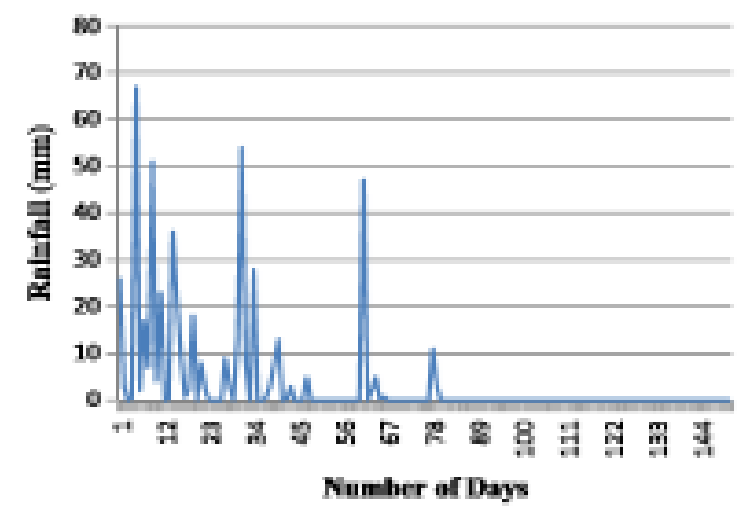

Figura 20: Caída de lluvia durante el período de estudio 


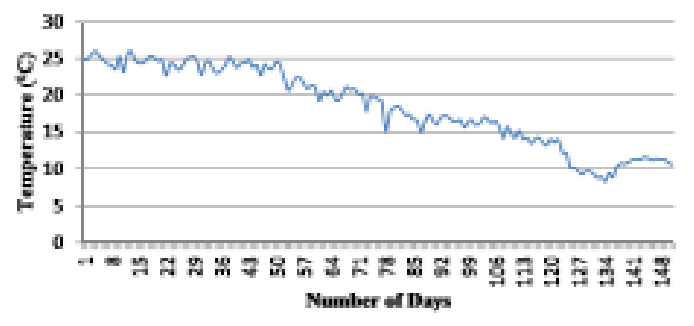

Figura 21: Variación de la temperatura durante el período de estudio

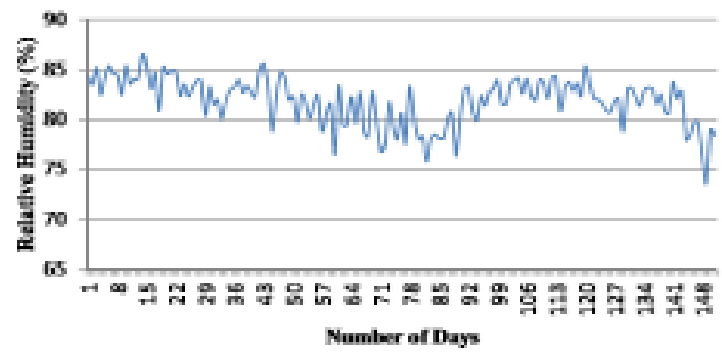

Figura 22: Humedad relativa promedio durante el período de estudio.

Con los datos tomados se formula el modelo de regresión que toma la forma de la siguiente ecuación:

$$
Y=A+\beta_{1} X_{1}+\beta_{2} X_{2}+\beta_{3} X_{3}+\beta_{4} X_{4}+\beta_{5} X_{5}+\beta_{6} X_{6}
$$

Donde:

$Y$ : Reducción de la eficiencia de los módulos fotovoltaicos sucios.

A: Coeficiente de regresión.

$X_{1}$ : Temperatura de la célula $\left({ }^{\circ} \mathrm{C}\right)$.

$X_{2}$ : Densidad de deposición de polvo $\left(\mathrm{g} / \mathrm{m}^{2}\right)$

$X_{3}:$ Humedad $(\%)$

$X_{4}:$ Radiación inclinada $\left(\mathrm{W} / \mathrm{m}^{2}\right)$.

$X_{5}:$ Lluvia $(\mathrm{mm})$.

$X_{6}$ : Temperatura ambiente $\left({ }^{\circ} \mathrm{C}\right)$

i) Los futuros sistemas de energía fiables y respetuosas del medio ambiente deben predecir la energía que pueden entregar de los recursos renovables. Sin embargo, la degradación del rendimiento inducido por el clima representa un gran inconveniente en los sistemas fotovoltaicos. Este trabajo propone una solución rentable personalizado que permite la monitorización y predicción del rendimiento de los sistemas fotovoltaicos, el fomento de estrategias de planificación y control de modo eficiente. Se propone utilizar un regulador seguidor del punto de máxima potencia, una estación meteorológica que incluya la medición de polvo, un sistema de adquisición de datos inalámbrica y una estación de monitoreo y registro basado en LabVIEW [28]

A modo de ejemplo, se muestra en las figuras (2327) la investigación del rendimiento del módulo fotovoltaico bajo las condiciones ambientales de Doha, usando un sistema de medición personalizada y monitoreada [28].

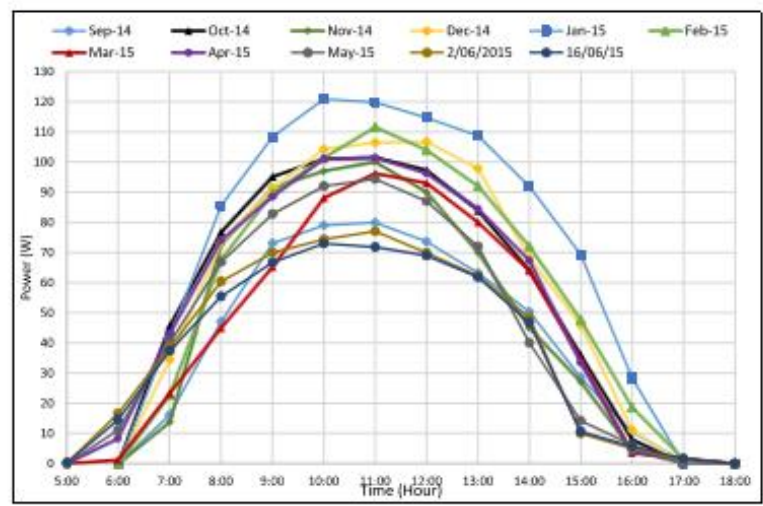

Figura 23: Salida de potencia de módulos fotovoltaicos de días típicos de setiembre 2014 a Junio del 2015 en Doha; bajo varias temperaturas, radiación solar y humedades relativas

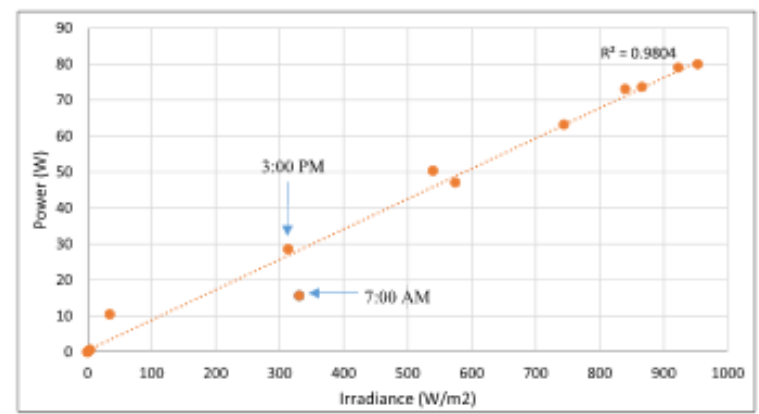

Figura 24: Potencia de salida versus radiación solar de módulos fotovoltaicos 


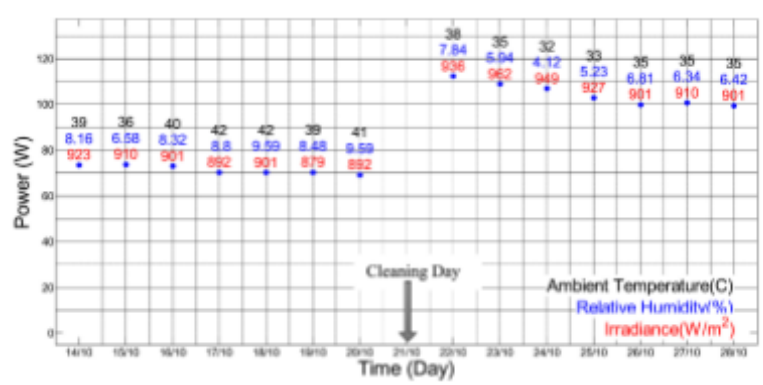

Figura 25: Datos para máxima potencia del módulo fotovoltaico antes y después de su limpieza durante Octubre del 2014

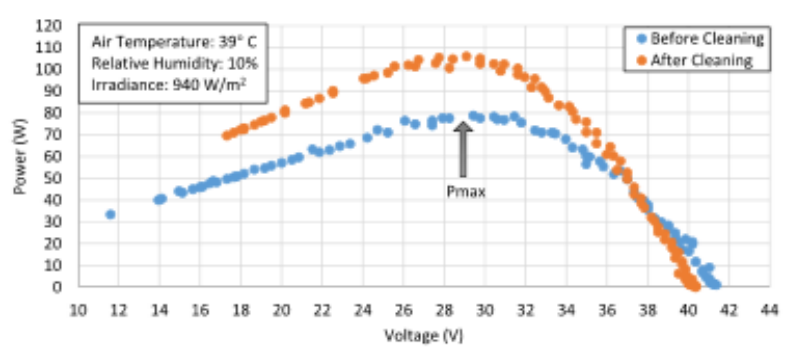

Figura 26: Curva P-V antes y después de la limpieza del módulo fotovoltaico en Octubre del 2014

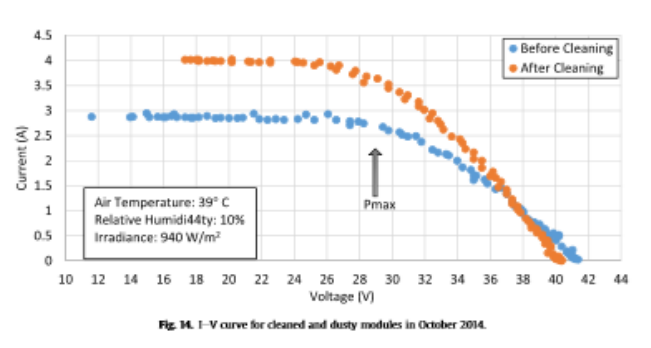

Figura 27: Curva I-V antes y después de la limpieza del módulo fotovoltaico en Octubre del 2014

\section{ARQUITECTURA DEL SISTEMA DE CONTROL PROPUESTO}

En la figura 28 se muestra la arquitectura del sistema de control propuesto.

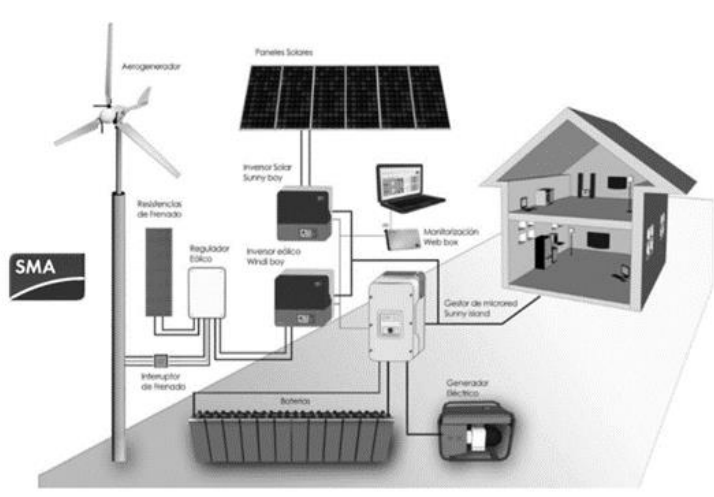

Figura 28: Smart Grid Aislado

Fuente: Manual de aerogeneradores ENAIR

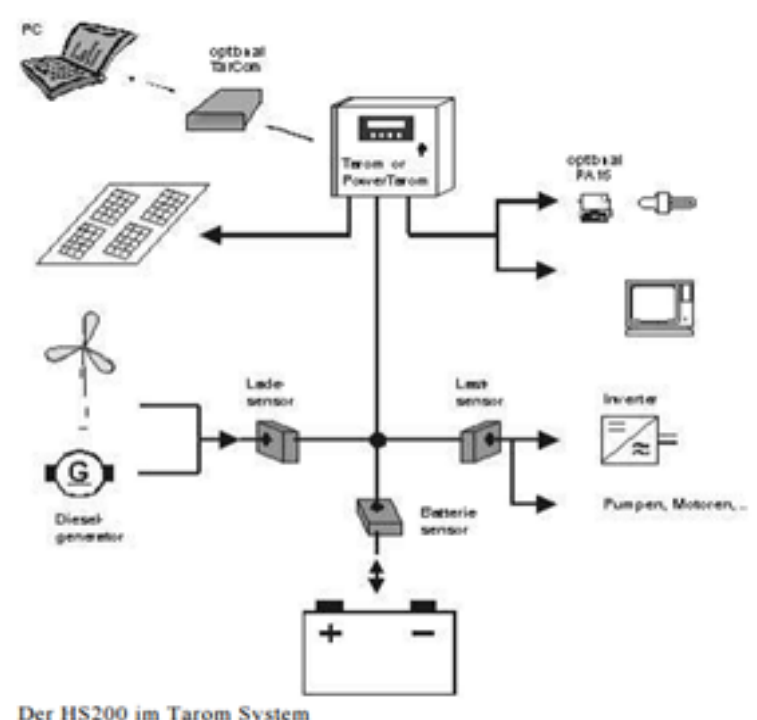

Figura 29: Ubicación de los instrumentos de medición

Fuente: Catálogo Steca
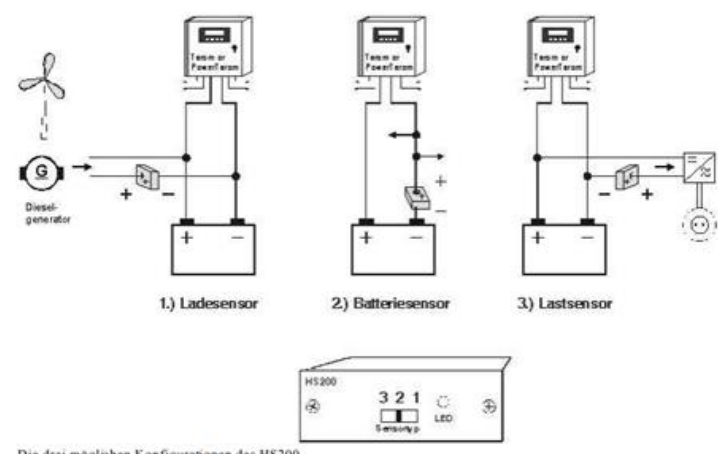

Figura 30: Tres posibilidades de configuración del HS200

Fuente: Catálogo Steca 
Durante la selección de la instrumentación para poder evaluar el funcionamiento de nuestro sistema fotovoltaico, se ha preferido usar la marca Steca por las bondades de funcionamiento, garantía de cinco años y lo más importante permite un monitoreo a distancia vía internet.

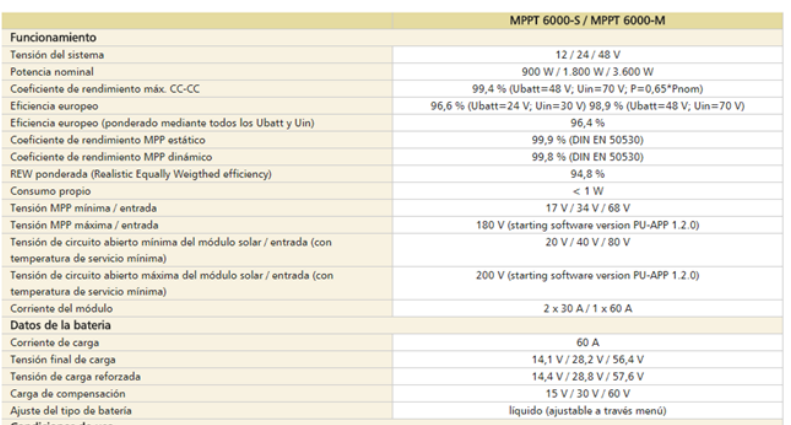

Figura 31: Características de los Reguladores de Carga Tarom MPPT 6000-S / MPPT 6000-M

Fuente: Catálogo Steca

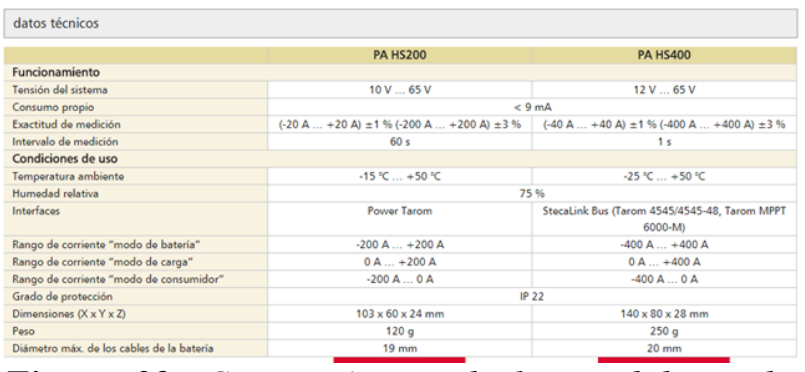

Figura 32: Características de los medidores de corriente PA HS200 / PA HS400

Fuente: Catálogo Steca

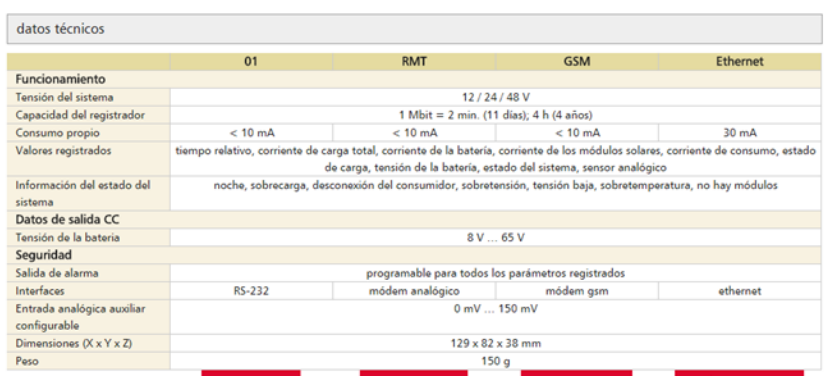

Figura 33: $\overline{\text { Características }}$ del Registrador de datos PA Tarcom GSM

Fuente: Catálogo Steca
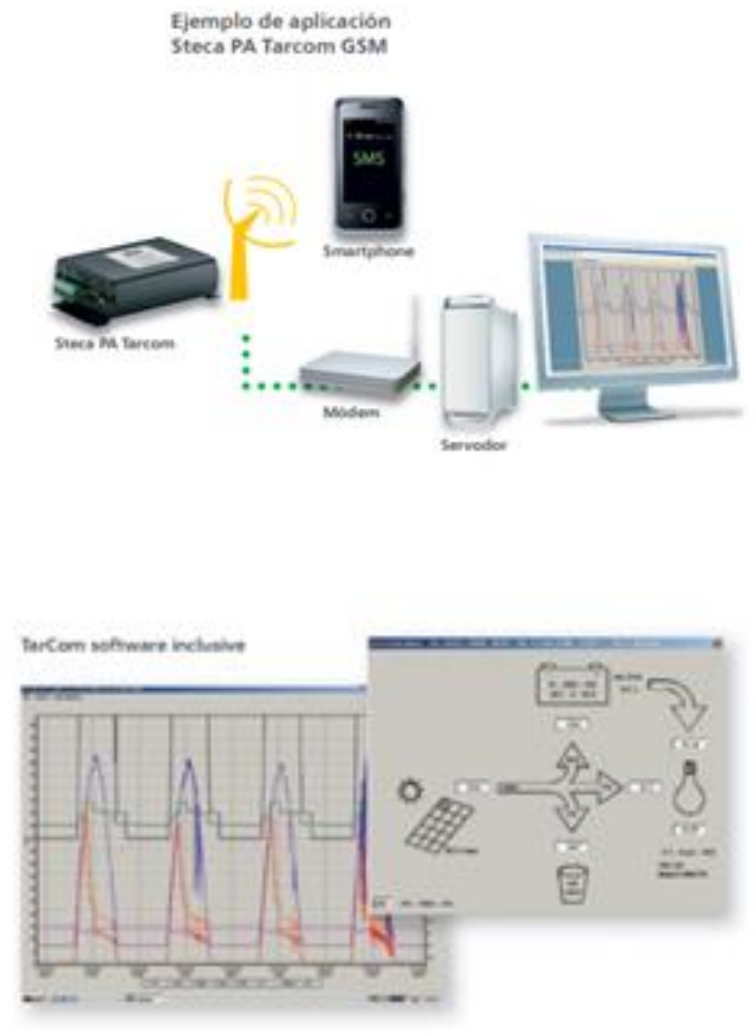

Figura 34: Ejemplo de aplicación del Registrador de datos PA Tarcom GSM

Fuente: Catálogo Steca

\section{CONCLUSIONES}

Los sistemas de energía fotovoltaico están siendo utilizados para el suministro de energía eléctrica en áreas urbanas, rurales y remotas habiendo logrado superar la intermitencia de los recursos de la energía solar.

Se propone desarrollar una metodología para la optimización del diseño y operación de sistemas fotovoltaicos, instalados en zonas rurales en el Perú. En ese contexto, contar con el desarrollo de una metodología para la optimización de sistemas fotovoltaicos autónomos a instalarse en el Perú, utilizando el criterio de red eléctrica inteligente, creará confianza en los inversionistas y beneficiará al usuario.

El uso de energías renovables hace posible el aprovechamiento de recursos naturales en forma sostenible, reduce el consumo de combustibles fósiles, no genera emisiones efecto invernadero, ni residuos sólidos y lleva a un mejoramiento de la calidad del medio ambiente. 


\section{REFERENCIAS}

1 Huang, Y., Quibria, M.G., 2013. Green growth: theory and evidence, world institute for development economics research, Working Paper No. 2013/056.

2 Jänique, M., 2012. Green growth: from a growing eco-industry to economic sustainability. Energy Policy 48, 13-21.

3 Hallegate, S., Heal, G., Fay, M., Treguer, D., 2012. From growth to green growth - a framework, the national bureau of economic research, Working Paper No. 17841.

4 Toman, M. 2012. Green growth: an exploratory review. WB Policy Research Working Paper 6067. World Bank.

5 Brown, M.A., Southworth, F., 2008. Mitigating climate change through green buildings and smart growth. Environ. Planning 40 (3), 653-675.

6 Gill, S.E., Handley, J.F., Ennos, A.R., Pauleit, S., 2007. Adapting cities for climate change: the role of the green infrastructure. Built Environ. 33 (1), 115-133.

7 Rycrofta, M.J., Israelssonb, S., Pricec, C., 2000. The global atmospheric electric circuit, solar activity and climate change. J. Atmos. Solar-Terrestrial Phys. 62 (17-18), 15631576.

8 Wagle, A.M., Lobo, A.M., Santosh Kumar, A., Shubhangi Patil, 2008. Real time webbased condition monitoring system for power transformers - case study. International Conference on Condition Monitoring and Diagnosis, Beijing.

9 Trovao, J.P., Santos, F.M., Silva, M.J., Jorge, H.M., 2008. A web-based monitoring approach for power systems in industrial plants. IEEE International Symposium on Industrial Electronics, Cambridge, pp. 17691774.

10 Apostolos Meliones a, Spyros Apostolacos ${ }^{\mathrm{b}}$, Angeliki Nouvaki ${ }^{\mathrm{a}}$. A web-based threetier control and monitoring application for integrated facility management of photovoltaic systems. ${ }^{a}$ Department of Digital Systems, University of Piraeus, 80 Dimitriou and Karaoli Str., GR-18534, Greece. ${ }^{\mathrm{b}}$ in Access, R\&D Center, 12 Sorou Str., 15125 Maroussi, Athens, Greece. 2014.
Kastner, W., Neugschwandtner G., Soucek S., Newman H.M, 2005.

Communication systems for building automation and control. In: Proceedings of the IEEE, Special Issue on Industrial Communication Systems, 93 (6), pp. 1178 1203.

13 Dorf, R.C., Bishop, R.H., 2010. Modern Control Systems, twelveth ed. Prentice Hall, ISBN-13: 978-0136024583.

14 Sinha S,ChandelSS, 2014. Review of software tools for hybrid renewable energy systems.RenewableSustainableEnergyRev20 14; 32:192-205.

15 Zhou W, Lou C, Li Z, Lu L, Yang H. 2010. Current status of research on optimum sizing of stand-alone hybrid solar-wind power generation systems. Appl Energy; 87:380-9.

16 Erdinc O, Uzunoglu M. 2012. Optimum design of hybrid renewable energy systems: overview of different approaches. Renewable Sustainable Energy Rev; 16:1412-25.

17 Fadaee M, MAM. Radzi. 2012. Multiobjective optimization of a stand-alone hybrid renewable energy system by using evolutionary algorithms: a review. Renewable Sustainable Energy Rev; 16:3364-9.

18 Luna-Rubio R, Trejo-Peres M, VargasVazquez D,Ríos-Moreno GJ 2012. Optimal sizing of renewable hybrids energy systems: are view of methodologies. Sol Energy; 86:1077-88.

19 Khatib T, Mohamed A, Sopian K 2013. A review of photovoltaic systems size optimization techniques. Renewable Sustainable EnergyRev; 22:454-65.

20 Upadhyay S, Sharma MP 2014. A review on configurations, control and sizing methodologies of hybrid energy systems. Renewable Sustainable Energy Rev; 38:4763.

21 Chauhan A, Saini RP 2014. A review on integrated renewable energy system based power generation for stand-alone applications: configurations, storage options, sizing methodologies and control. 
Renewable Sustainable Energy Rev; 38:99120.

22 Bourennani F, Rahnamayan S,

NatererGF. 2015. Optimal design methods for hybrid renewable energy systems. Int $\mathbf{J}$ Green Energy; 12:148-59.

23 Bhandari B, Lee KT, Lee GY, Cho YM, Ahn SH. 2015. Optimization of hybrid renewable energy power systems: a review. Int J Precis Eng Manuf Green Technol: 2(1): 99-112.

24 Bilal BO, Nourou D, Kébé CMF, Sambou V, Ndiaye PA, NdongoM. 2015. Multiobjective optimization of hybrid $\mathrm{PV} /$ wind/diesel/battery systems for decentralized application by minimizing the levelized cost of energy and the $\mathrm{CO} 2$ emissions. Int J Phys Sci10 (5):192-203.

25 Nogueira CEC, Vidotto ML, Niedzialkoski RK, Melegaride Souza SN, Chaves LI, Edwiges T, et al. 2014. Sizing and simulation of a photovoltaic-wind energy system using batteries, applied for a small rural property located in the south of Brazil. Renewable Sustainable Energy 29:151-7.

26 Luigi Fortuna, Guiseppe Nunnari, Silvia Nunnari. 2016. A new fine-grained classification strategy for solar daily radiation patterns. Dipartimento di Ingegneria Elettrica ed Informatica, Universita degli Studi di Catania, Viale A. Doria 6, Catania 95125, Italy.

27 Basant Raj Paudyal, Shree Raj Shakya. 2016. Dust accumulation effects on efficiency of solar PV modules for off grid purpose: A case study of Kathmandu. Department of Mechanical Engineering, Institute of Engineering, Tribhuvan University, Kathmandu, Nepal.

28 Farid Touati, M.A. Al Hitmi, Noor Alam Chowdhury, Jehan Abu Hamad, Antonio J.R. San Pedro Gonzales. 2015.

Investigation of solar PV performance under Doha weather using a customized measurement and monitoring system. Department of Electrical Engineering, Qatar University, Qatar.

Correspondencia omorales@uni.edu.pe 